\title{
Coherent control of a nitrogen-vacancy center spin ensemble with a diamond mechanical resonator: supplementary material
}

\author{
E. R. MacQuarrie, T. A. Gosavi, A. M. Moehle, N. R. Jungwirth, \\ S. A. BHAVE, AND G. D. FUCHS
}

Cornell University, Ithaca, New York 14853, USA

*Corresponding author: gdf9@cornell.edu

Published 5 March 2015

\begin{abstract}
This document provides supplementary information to "Coherent control of a nitrogen-vacancy center spin ensemble with a diamond mechanical resonator," http://dx.doi.org/10.1364/optica.2.000233. We include a description of the stress coupling constant derivation, a list of device fabrication improvements, additional details of the Rabi and Ramsey measurements presented in the main text, and magnetic Hahn echo measurements of the homogeneous dephasing time $\boldsymbol{T}_{\mathbf{2}}$. ( 2015 Optical Society of America
\end{abstract}

http://dx.doi.org/10.1364/optica.2.000233.s001

\section{NV CENTER STRESS COUPLING}

Ovartchaiyapong, et al measured the NV center strain coupling to be $d_{\perp} / 2 \pi=21.5 \mathrm{GHz} /$ strain and $d_{\|} / 2 \pi=13.3 \mathrm{GHz} /$ strain for perpendicular and axial strain, respectively [S1]. Since our mechanical resonator generates acoustic waves by applying a pressure to one face of the diamond crystal, we choose to work in units of stress. To convert the measured constants from strain to stress, we first rotate the measured couplings from the coordinate system defined by the NV center to the lattice coordinates. We then use the stiffness matrix for diamond [S2]

$$
\left(\begin{array}{c}
\sigma_{x x} \\
\sigma_{y y} \\
\sigma_{z z} \\
\sigma_{y z} \\
\sigma_{z x} \\
\sigma_{x y}
\end{array}\right)=\left(\begin{array}{cccccc}
C_{11} & C_{12} & C_{12} & 0 & 0 & 0 \\
C_{12} & C_{11} & C_{12} & 0 & 0 & 0 \\
C_{12} & C_{12} & C_{11} & 0 & 0 & 0 \\
0 & 0 & 0 & C_{44} & 0 & 0 \\
0 & 0 & 0 & 0 & C_{44} & 0 \\
0 & 0 & 0 & 0 & 0 & C_{44}
\end{array}\right)\left(\begin{array}{c}
\epsilon_{x x} \\
\epsilon_{y y} \\
\epsilon_{z z} \\
\epsilon_{y z} \\
\epsilon_{z x} \\
\epsilon_{x y}
\end{array}\right)
$$

to convert strain/GHz into $\mathrm{GPa} / \mathrm{GHz}$ (stress/GHz). The elastic constants $C_{i j}$ are given in Table S1. Finally, we rotate back into the coordinates of the NV center to find the stress coupling constants $\epsilon_{\perp} / 2 \pi=0.015 \mathrm{MHz} / \mathrm{MPa}$ and $\epsilon_{\|} / 2 \pi=$ $0.012 \mathrm{MHz} / \mathrm{MPa}$ used in the main text.

\section{DEVICE IMPROVEMENTS}

A number of refinements in device fabrication have improved the performance of the HBAR devices presented in this work

\begin{tabular}{c|c|c}
$C_{11}$ & $C_{12}$ & $C_{44}$ \\
\hline $1076.4 \mathrm{GPa}$ & $125.2 \mathrm{GPa}$ & $577.4 \mathrm{GPa}$
\end{tabular}

Table S1. Stiffness constants for diamond[S3].

over the device discussed in Ref. [S4], enabling coherent spin control driven with a mechanical resonator. Our most impor-tant advance was to develop in-house growth of piezoelectric $\mathrm{ZnO}$ for use with the HBAR transducer to replace the foundry grown piezoelectric AlN used in Ref. [S4]. Although the two films perform comparably, the faster fabrication time of a fully local process enabled rapid device development. We were able to engineer a better impedance match between the HBAR and the microwave driving circuit. Additionally, as pictured in Fig. S1, we apodized the shape of the HBAR top contact to inhibit lat-eral modes. These modes drain power from the device without contributing to the stress wave of interest. Thus, by apodizing the top contact, we increased the efficiency of the HBAR. Addi-tionally, we changed the geometry of the back-side microwave antenna, minimizing its contact with the shadow of the HBAR active region. Combined, these small optimizations and cleaner fabrication generated higher quality devices with better power coupling to the microwave circuit. 

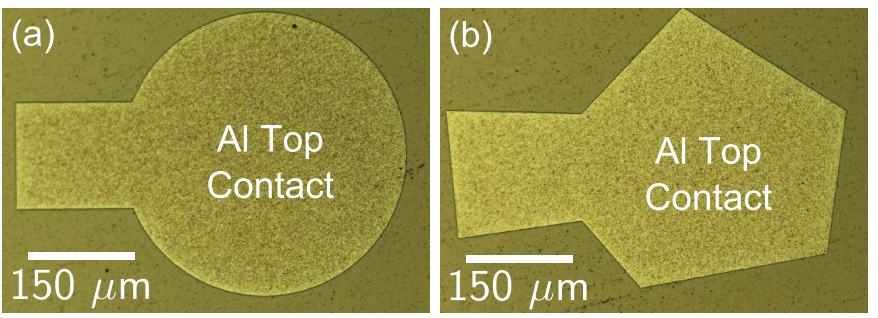

Fig. S1. (a) A circular HBAR as was used in [S4]. (b) An apodized HBAR design as used in the current work.

\section{MECHANICAL RABI MEASUREMENTS}

\section{A. Readout Through $|+1\rangle$}

As a control, we performed a second type of Rabi measurement. In this alternative pulse sequence, after optically pumping the $\mathrm{NV}$ center into $|0\rangle$ we once again apply a magnetic $\pi$-pulse to resonantly move the population from $|0\rangle$ to $|-1\rangle$. We then pulse the resonant mechanical driving field for a length $\tau$ to drive the $|-1\rangle \leftrightarrow|+1\rangle$ transition. Finally, we use a magnetic adiabatic passage to robustly transfer the population that was driven into $|+1\rangle$ to $|0\rangle$ where we read out the spin state optically. This differs from the Rabi measurement presented in the main text in that we extract population from $|+1\rangle$, not $|-1\rangle$, for optical readout.

Fig. S2 shows the results of this measurement plotted alongside a mechanically driven Rabi measurement that uses a magnetic adiabatic passage to transfer population from $|-1\rangle$ to $|0\rangle$ after the mechanical Rabi pulse. Both of these measurements were done on Sample A. As expected, the results are nearly identical. The difference in amplitudes comes from fidelity differences between the $|+1\rangle \leftrightarrow|0\rangle$ and $|0\rangle \leftrightarrow|-1\rangle$ magnetic pulses.

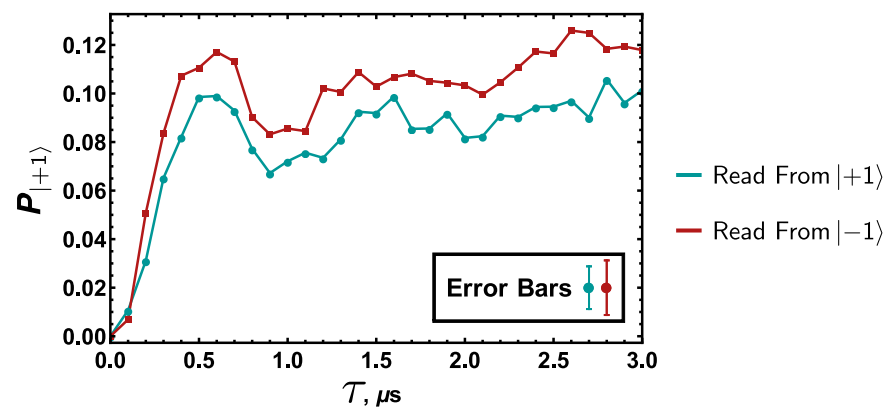

Fig. S2. Mechanically driven Rabi oscillations as read out from the $|+1\rangle$ (blue) and $|-1\rangle$ (red) spin states. These measurements were performed on Sample A.

\section{B. Mechanical Rabi Sequence for Sample B}

Fig. S3a shows the mechanical Rabi oscillations plotted in Fig. 3b of the main text. This measurement was taken by sweeping a pair of magnetic $\pi$-pulses through a fixed-length mechanical pulse. To further elucidate this pulse sequence, Fig. S3b provides a snapshot of the pulse sequence at each of the notable points indicated by dashed lines in Fig. S3a and described in the figure caption.
We model the ringing of a normalized mechanical driving field with the functions $1-e^{-\frac{t}{\tau_{r}}}$ for ring-up and $e^{-\frac{t-t_{0}}{\tau_{r}}}$ for ringdown where $t_{0}=L+\tau_{r} \log \left(1-e^{-\frac{t}{t^{t}}}\right.$ and $\tau_{r}=2 Q / \omega_{m}$ [S5]. These functions allow us to compute the mechanical pulse area enclosed between the two magnetic $\pi$-pulses for each value of $\tau$. Fig. S3b plots this normalized Rabi interval as a function of $\tau$.

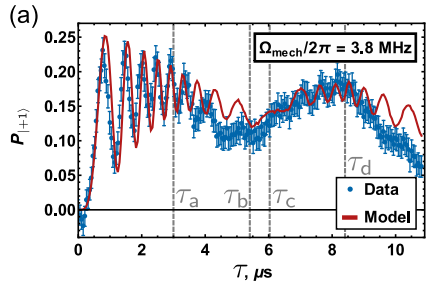

(c)
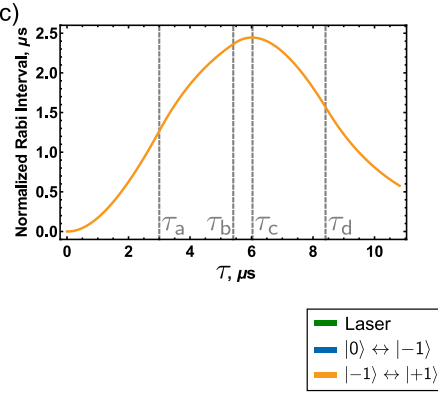

(b) $\tau=0$

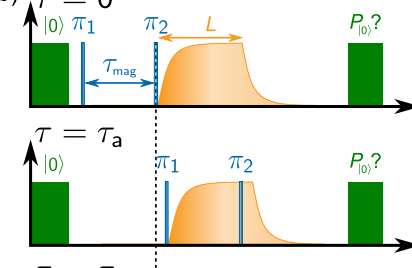

$\tau=\tau_{\mathrm{b}}$

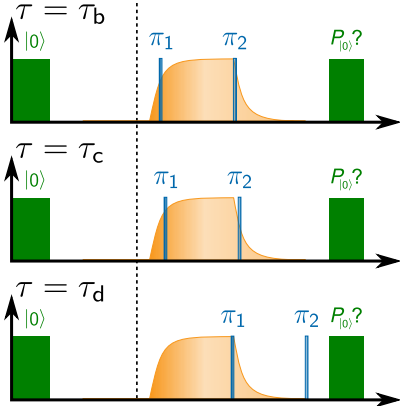

Fig. S3. (a) Rabi oscillations driven mechanically with a high $Q$ mechanical resonator. From left to right, the dashed lines correspond to $\pi_{2}$ entering the ring down portion of the mechanical pulse, $\pi_{1}$ entering the ring up, the maximum mechanical pulse area $\tau_{c}$, and $\pi_{1}$ entering the ring down. (b) Pulse sequence at each of the notable times labeled in (a) and (c). (c) Mechanical pulse area enclosed between the two magnetic $\pi$ pulses as a function of $\tau$. For a mechanical pulse normalized to its amplitude after ring up, this pulse area corresponds to the normalized Rabi interval.

\section{Mechanical Rabi Model for Sample B}

To fit the mechanical Rabi data shown in Fig. $3 \mathrm{~b}$ of the main text, we solve the Schrödinger equation to find the population in $|+1\rangle$ after applying the relevant portion of an $L=3 \mu$ s mechanical pulse. We use the Hamiltonian

$$
H_{u p}=\left(\begin{array}{ccc}
\delta & 0 & \frac{1}{2} \Omega(z)\left(1-e^{-\frac{t}{\tau_{r}}}\right) \\
0 & 0 & 0 \\
\frac{1}{2} \Omega(z)\left(1-e^{-\frac{t}{\tau_{r}}}\right) & 0 & -\delta
\end{array}\right)
$$

when the resonator is ringing up and the Hamiltonian

$$
H_{\text {down }}=\left(\begin{array}{ccc}
\delta & 0 & \frac{1}{2} \Omega(z) e^{-\frac{t-t_{0}}{\tau_{r}}} \\
0 & 0 & 0 \\
\frac{1}{2} \Omega(z) e^{-\frac{t-t_{0}}{\tau_{r}}} & 0 & -\delta
\end{array}\right)
$$

when the resonator is ringing down. Quasi-static magnetic bath noise takes the form of a randomized detuning $\delta$ drawn from a Gaussian distribution with a standard deviation $\sigma=\sqrt{2} / T_{2}{ }^{*}[\mathrm{~S} 6]$. The magnetic Ramsey measurement shown in Fig. S4 sets $T_{2}^{*}=$ $0.68 \mu \mathrm{s}$. 


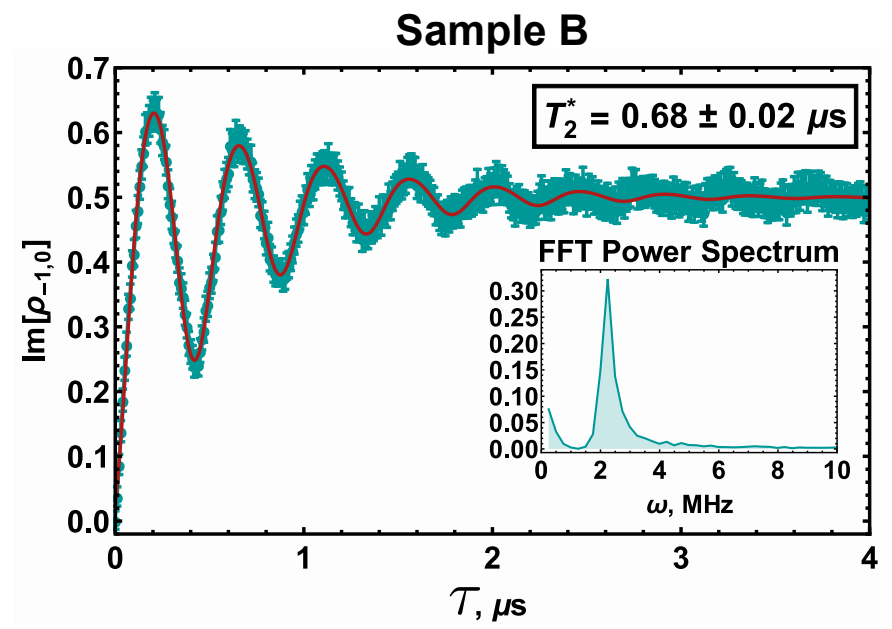

Fig. S4. Magnetic Ramsey measurement of $T_{2}^{*}$ for Sample B in the $\{0,-1\}$ subspace.

Defining the result of this computation as the function $f(\tau, \Omega(z))$, we then perform a spatially-weighted average over the point spread function (PSF) of our confocal microscope to account for spatial inhomogeneities in our mechanical driving field. The resulting signal takes the form

$$
P_{|+1\rangle}=\frac{C}{\int_{0}^{\infty} g\left(z, z_{0}\right) \mathrm{d} z} \int_{0}^{\infty} g\left(z, z_{0}\right) f(\tau, \Omega(z)) \mathrm{d} z
$$

where $C$ accounts for partial polarization of the nuclear spin sublevel, $\Omega(z)=\Omega_{\text {mech }}\left|\sin \frac{2 \pi z}{\lambda_{B}}\right|$ is the mechanical driving field, $\lambda_{B}$ is the wavelength of the stress wave, and $g\left(z, z_{0}\right)$ describes a Gaussian approximation to a PSF centered at the focal depth $z_{0}$ with a depth dependent FWHM as described in Ref. [S4]. To produce the model curve in Fig. $3 \mathrm{~b}$ of the main text, we used the parameters $\Omega_{\text {mech }} / 2 \pi=3.8 \mathrm{MHz}, z_{0}=5.9 \mu \mathrm{m}, C=0.414$ (measured with mechanically driven spin resonance), and $\lambda_{B}=$ $29.6 \mu \mathrm{m}$. The simulation was repeated 200 times, and these results were averaged to produce the final curve.

\section{Depth Dependent Mechanical Rabi Simulations}

In Fig. S5, we use the model developed in Section C to simulate mechanical Rabi measurements taken at various depths inside the diamond substrate. The simulations, which are not fits to any data, correctly reproduce the experimentally observed decrease in the visibility of the Rabi oscillations for measurements taken as the focal depth approaches a node of the stress wave. Because the inhomogeneities in the mechanical driving field are largest near a stress wave node, the spins within our collection volume dephase more quickly near a node, reducing the visibility of the Rabi oscillations.

The simulation also reproduces the limited sensitivity in the frequency of the Rabi oscillations to the measurement depth. This limited sensitivity arises in part because, within the finite collection volume of our microscope, the NV centers that experience the largest Rabi field will be the best protected against dephasing. The visibility of these faster Rabi oscillations can thus be larger than the slower Rabi oscillations also present in the signal, even if the spins driven at slower Rabi frequencies are more optimally positioned within our collection volume. This apparent insensitivity of Rabi frequency to position also arises in part from purely geometric considerations, which we confirmed by separately performing simulations that do not account for decoherence.

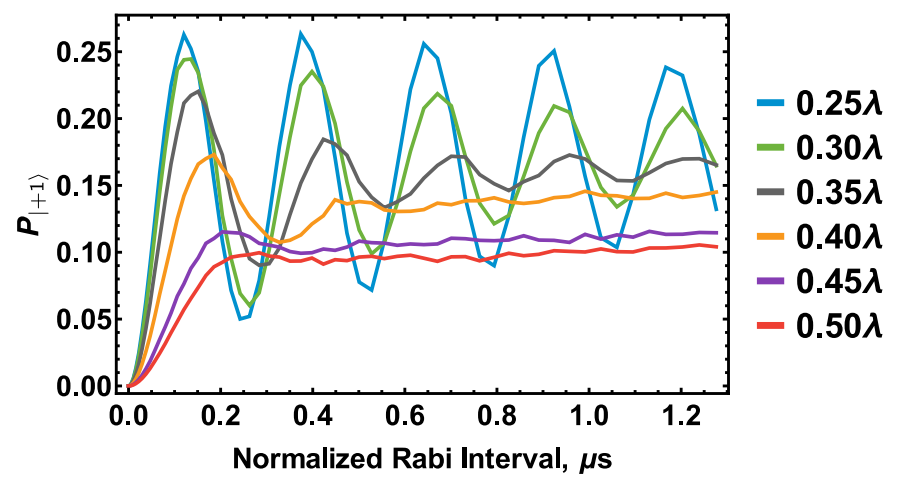

Fig. S5. Mechanical Rabi measurements simulated with the numerical model detailed in Section $C$ above for several different measurement depths. The depth is measured relative to the wavelength $\lambda$ of the stress wave.

\section{RAMSEY MEASUREMENTS}

\section{A. Ramsey Pulse Sequences}

Fig. S6 shows the pulse sequences used for the Ramsey measurements presented in the main text. To eliminate experimental artifacts, we modified the typical Ramsey measurement to include a second measurement for each data point. We first execute the typical $\pi / 2-\tau-\pi / 2$ Ramsey sequence. Immediately afterward, we perform a $\pi / 2-\tau-(-\pi / 2)$ sequence. The difference of these two measurements equals twice the imaginary portion of the qubit's coherence $\operatorname{Im}\left[\rho_{i, j}\right]\left(i, j \in\left\{\left(m_{s}=\right)+1,0,-1\right\}, i \neq j\right)$. We further modify the Ramsey sequence for the mechanically driven qubit by advancing the phase of the second $\pi / 2$-pulse by $\omega_{\text {rot }}\left(\tau+\tau_{\pi / 2}\right)$. This extra phase shift introduces a known periodicity to the measurement that aids visualization of the decay envelope. (a)

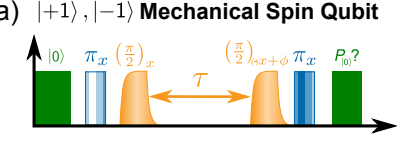

(c)

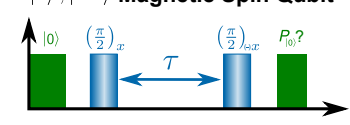

(b) $|+1\rangle,|-1\rangle$ Magnetic Spin Qubit

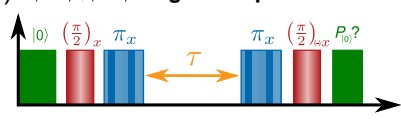

(d) $|+1\rangle,|0\rangle$ Magnetic Spin Qubit

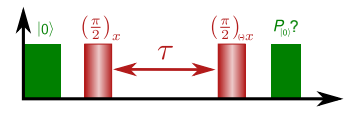

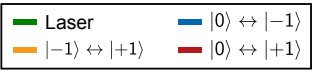

Fig. S6. Pulse sequences used for the Ramsey measurements presented in the main text.

\section{B. Ramsey Measurement Normalization}

Two measurements were used to normalize the spin contrast for the magnetic Ramsey measurements in the $\{+1,0\}$ and $\{0$, $-1\}$ subspaces. The maximum spin signal $y_{N P}$ is measured by optically pumping the NV center into $|0\rangle$, shuttering the laser for the fixed dark time in which no pulses were applied, 
and then reading out the NV center fluorescence. Applying a single magnetic $\pi$-pulse to the relevant qubit during that dark time gives the minimum spin signal $y_{\pi}$. Defining the $\pi / 2-$ $\tau-\pi / 2$ measurement results as $y_{+}$and the $\pi / 2-\tau-(-\pi / 2)$ measurement results as $y_{-}$, the expression

$$
\operatorname{Im}\left[\rho_{i j}\right]=\frac{1}{2} \frac{y_{+}-y_{-}}{y_{N P}-y_{\pi}}
$$

gives the normalized coherence of the $|i\rangle,|j\rangle$ qubit.

For the magnetic $\{-1,+1\}$ qubit Ramsey measurement, the same "no pulse" measurement gives the maximum spin signal $y_{N P}$. We define the minimum spin signal $y_{\pi}$ as the average of the signal from a single magnetic $\pi$-pulse on the $\{+1,0\}$ qubit and the signal from a single magnetic $\pi$-pulse on the $\{0,-1\}$ qubit.

For the mechanically driven $\{-1,+1\}$ qubit, the "no pulse" measurement once again sets the maximum spin signal for the mechanically driven $\{-1,+1\}$ qubit. The minimum spin signal is set by a $\pi_{m a g}-\pi_{m e c h}-\pi_{m a g}$ pulse sequence. Here, $\pi_{m a g}$ corresponds to a magnetic $\pi$-pulse on the $\{0,-1\}$ qubit, and $\pi_{m e c h}$ describes a mechanical $\pi$-pulse on the $\{-1,+1\}$ qubit.
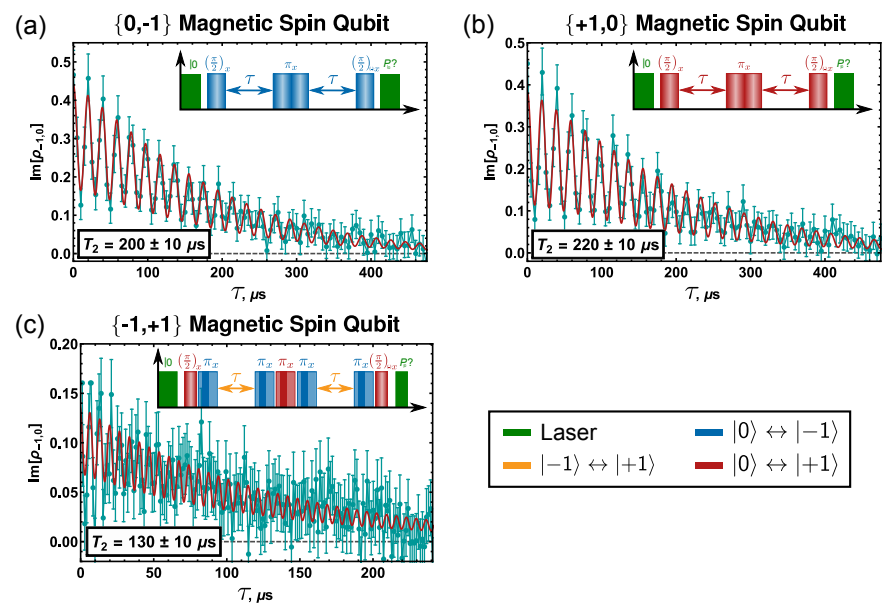

Fig. S7. Hahn echo data for (a) a magnetically driven $\{0,-1\}$ qubit, (b) a magnetically driven $\{+1,0\}$ qubit, and (c) a magnetically driven $\{-1,+1\}$ qubit. The pulse sequence for each measurement is inset within each plot.

\section{HAHN ECHO MEASUREMENTS}

We performed magnetic Hahn echo measurements of the homogeneous dephasing time $T_{2}$ in Sample A. We were unable to perform a mechanical Hahn echo experiment as intrinsic spin dephasing in our device limited the spin contrast after a mechanically driven $2 \pi$ nutation to the prohibitive value of $\approx 1 \%$. Fig. S7 shows the Hahn echo data for each magnetically driven qubit examined in the main text. Once again, we measure roughly twice the coherence for the $\{+1,0\}$ and $\{0,-1\}$ qubits when compared to the $\{-1,+1\}$ qubit.

\section{REFERENCES}

1. P. Ovartchaiyapong, K. W. Lee, B. A. Myers, and A. C. B. Jayich, "Dynamic strain-mediated coupling of a single diamond spin to a mechanical resonator," Nat. Commun. 5, 4429 (2014).

2. F. Irgens, Continuum Mechanics (Springer, 2008).
3. C. A. Klein and G. F. Cardinale, "Young's modulus and poisson's ratio of cvd diamond," Diam. Relat. Mater. 2, 918-923 (1993).

4. E. R. MacQuarrie, T. A. Gosavi, N. R. Jungwirth, S. A. Bhave, and G. D. Fuchs, "Mechanical spin control of nitrogenvacancy centers in diamond," Phys. Rev. Lett. 111, 227602 (2013).

5. W. M. Seibert, Circuits, Signals, and Systems (The MIT Press, 1985).

6. C. D. Aiello, M. Hirose, and P. Cappellaro, "Composite-pulse magnetometry with a solid-state quantum sensor," Nat. Commun. 4, 1419 (2013). 